\title{
III. Geburtshülflich-gynäkologische Mitteilungen aus anderen
} Gesellschaften und Vereinen

I. Verein $\Gamma$ reiburge $\Gamma$ Ärzte.

(Nach Munch, med. Wochenschr.)

25. Juni. Krönig: Ergebnis der Diskussíon über die Behandlung des Kindbettfiébers auf dem letzten Gynäkologenkongresse. Bericht fehlt.

II. Medizinische Gesellschaft in Giessen.

(Nach Deutsche med. Wochenschr.)

$l_{i}>$. Juni. Rühl: Zerreissung der hinteren Scheidenwand während der Geburt. Cfr.

Originalarbeiten im Literaturbericht.

Rühl: Demonstration von Fäden, die vor langer Zeit in die Bauchhöhle versenkt worden ivaren. Franqué: Demonstrationen: Kongenitaler Nabelbruch, durch Operation am Tage der Geburt geheilt. Prolaps des Netzes. Karzinom des S romanum per vaginam entfernt.

Sitzenfrey: Demonstrationen: Miliare submukõse Myome, ein Beitrag zn den sogenannten unstillbaren Blutungen des Uterus. Multiple Myom-büdungen der Uterusvenen.

III. Verein der Ärzte in Halle.

(Nach Munch, med. Wochenschr. 49.) 27. Oktober. Mohr spricht über neue Versuche über die Organhämolyse, speziell in der Placenta. Die Ergebnisse werden noch weiter ausführlich veröffentlicht.

IV. Klinische Demonstrationsabende der städtisehen Krankenanstalten

zu Dortmund.

(Nach Medizinische Klinik. 42.) 18. Oktober. Engelmann bespricht die Behandlung der chronischen $\Lambda$ dnexitis durch einen neuen Heissluftapparat.

V. Äeademia delle scienze mediehe di Palermo.

(La Ginecologia. Oktober.)

18. Juli. Montuoro: Fibroma occludente lo scavo. Fissed labour. Albuminuría. Feto putrífatio. Operazione cesarea Porro Guarígione. 